\title{
A numerical simulation of viscous shear effects on porous squeeze-film using the Darcy-Brinkman model
}

\author{
Mohamed Nabhani ${ }^{1, a}$, Mohamed El Khlifi² ${ }^{2}$ And BenyebKa Bou-SAÏD ${ }^{3}$ \\ 1 Université de Lyon, CNRS INSA-Lyon, LaMCoS, UMR 5259, 69621 Villeurbanne Cedex, France \\ 2 University Hassan II Mohammedia - Casablanca, Faculty of Sciences and Technics, BP 146, 20650 Mohammedia, Morocco \\ 3 Université de Lyon, CNRS INSA-Lyon, LaMCoS, UMR 5259, 20 avenue Albert Einstein, 69621 Villeurbanne Cedex, France
}

Received 2 February 2010, Accepted 14 June 2010

\begin{abstract}
In recent decades, problems related to the squeeze of fluid films in the presence of a porous medium draw attention of researchers and are the subject of many applied studies for industry and biomechanics. Our concerns in this paper are the numerical simulation of the viscous shear stresses effects on the fluid film characteristics between two discs with one porous. This study is based on the coupling, at the fluid film-porous disc interface, of the Darcy-Brinkman equations in the porous medium and the modified Reynolds equation describing the flow in the fluid film. The system of equations obtained is discretized by the means of finite differences method and solved numerically using the technique of Successive OverRelaxation (SOR). The results show that the viscous shear effects increase the radial and the axial fluid film velocities as well as the squeeze film velocity but decrease the response time. Moreover, these effects are enlarged for smaller viscous shear parameter and for smaller fluid film thickness.
\end{abstract}

Key words: Porous medium / Darcy-Brinkman model / squeeze-film / modified Reynolds equation / numerical simulation

Résumé - Étude numérique des effets visqueux sur l'écrasement d'un film en présence d'un milieu poreux par le modèle de Darcy-Brinkman. Ces dernières décennies, les problèmes liés à l'écrasement de films fluides en présence d'un milieu poreux attirent l'attention des chercheurs et font l'objet de nombreux travaux d'application dans l'industrie et la biomécanique. Nous nous intéressons dans cet article à la simulation numérique des effets des tensions visqueuses sur les caractéristiques de l'écrasement d'un film fluide entre deux disques dont l'un est poreux. Cette étude est basée sur le couplage, à l'interface film fluide - disque poreux, entre les équations de Darcy-Brinkman dans le milieu poreux et l'équation de Reynolds modifiée décrivant l'écoulement dans le film fluide. Le système d'équations obtenu est discrétisé par la méthode des différences finies et résolu numériquement par la technique de sur-relaxation. Les résultats obtenus montrent que les tensions visqueuses augmentent les vitesses radiales et axiales du film fluide et la vitesse d'écrasement, mais diminuent le temps d'écrasement. De plus, ces effets deviennent plus importants pour les plus petites valeurs du paramètre des tensions visqueuses et pour les faibles épaisseurs du film fluide.

Mots clés : Milieu poreux / modèle de Darcy-Brinkman / écrasement de film / équation de Reynolds modifiée / simulation numérique

\section{Introduction}

The porous squeeze-film problems have received great interests because of their wide areas of application in engineering practices such as machine tools, dampers and human joints. The term squeeze film applies to any two

\footnotetext{
${ }^{a}$ Corresponding author: nabhanimohamed@yahoo.fr
}

lubricated solids moving towards each other in order to move a viscous lubricant between them. When the two lubricated solids approach each other, the fluid film is partly squeezed and some of the fluid flows out through the porous medium. In the literature, several researchers have proposed approaches to investigate the squeeze film behaviour in the presence of a porous facing. 


\section{Nomenclature}

\begin{tabular}{|c|c|}
\hline $\mathrm{d} h / \mathrm{d} t$ & squeezing velocity $\left(\mathrm{m} \cdot \mathrm{s}^{-1}\right)$ \\
\hline$h$ & fluid film thickness $(\mathrm{m})$ \\
\hline$h_{0}$ & initial fluid film thickness (m) \\
\hline$H$ & porous disc thickness $(\mathrm{m})$ \\
\hline$D_{\mathrm{p}}$ & characteristic pore diameter $(\mathrm{m})$ \\
\hline$k$ & permeability $\left(\mathrm{m}^{2}\right)$ \\
\hline$p, \bar{p}$ & fluid film pressure (Pa.s), dimensionless fluid film pressure \\
\hline$p^{*}, \bar{p}^{*}$ & fluid pressure in the porous disc (Pa.s), dimensionless fluid pressure in the porous disc \\
\hline$r, \bar{r}$ & radial coordinate $(\mathrm{m})$, dimensionless radial coordinate \\
\hline$R$ & discs radius $(\mathrm{m})$ \\
\hline$R_{\mathrm{ep}}$ & Reynolds number in the porous medium \\
\hline$u, \bar{u}$ & radial velocity component in the fluid film $\left(\mathrm{m} \cdot \mathrm{s}^{-1}\right)$, dimensionless radial velocity component in the fluid film \\
\hline$u^{*}, \bar{u}^{*}$ & $\begin{array}{l}\text { radial velocity component in in the porous disc }\left(\mathrm{m} . \mathrm{s}^{-1}\right) \text {, dimensionless radial velocity component } \\
\text { in the porous disc }\end{array}$ \\
\hline$u_{0}^{*}, \bar{u}_{0}^{*}$ & $\begin{array}{l}\text { radial velocity component at the porous interface }\left(\mathrm{m} . \mathrm{s}^{-1}\right) \text {, dimensionless radial velocity component } \\
\text { at the porous interface }\end{array}$ \\
\hline$w, \bar{w}$ & axial velocity component in the fluid film $\left(\mathrm{m} . \mathrm{s}^{-1}\right)$, dimensionless axial velocity component in the fluid film \\
\hline$w^{*}, \bar{w}^{*}$ & axial velocity component in the porous disc $\left(\mathrm{m} . \mathrm{s}^{-1}\right)$, dimensionless axial velocity component in the porous disc \\
\hline$w_{0}^{*}, \bar{w}_{0}^{*}$ & $\begin{array}{l}\text { axial velocity component at the porous interface }\left(\mathrm{m} \cdot \mathrm{s}^{-1}\right) \text {, dimensionless axial velocity component } \\
\text { at the porous interface }\end{array}$ \\
\hline$W, \bar{W}$ & load capacity $(\mathrm{N})$, dimensionless load capacity \\
\hline$z, \bar{z}$ & axial coordinate $(\mathrm{m})$, dimensionless axial coordinate \\
\hline$\mu$ & dynamic viscosity of the fluid (Pa.s) \\
\hline$\mu^{*}$ & effective viscosity of the fluid in the porous medium (Pa.s) \\
\hline$\alpha$ & viscosity ratio, $\left(\mu^{*} / \mu\right)$ \\
\hline$\rho$ & density of the fluid $\left(\mathrm{kg} \cdot \mathrm{m}^{-3}\right)$ \\
\hline$\phi$ & porosity of the porous disc \\
\hline
\end{tabular}

Based on a linear biphasic mixture theory for the transport of fluid in deformable porous media under loading, many authors investigated the poroelastic squeeze flow. The performance of squeeze flow of non Newtonian fluid between an infinitely long cylinder and a poroelastic surface and between an impermeable rigid plate and a poroelastic thin layer with reference to synovial joints was analysed by Bujurke et al. [1-4]. The non Newtonian squeeze flow for two poroelastic plates representing human knee joint was analysed by Tandon et al. [5]. In a series of papers, Hlavaceck [6-10] presented a general analysis of an axially symmetric squeeze film for two poroelastic thin layers modelling the human articular cartilage. Note that all the above mentioned studies treated the poroelastic material as a mixture of two interacting continua: a linear elastic solid and an ideal fluid. So, the effects of viscous shear stresses have not been considered. Accounting for the boundary layer effects by assuming the interstitial fluid in the porous medium to be Newtonian with an apparent viscosity, Shou et al. [11] analysed the axisymmetric squeeze flow of a Newtonian fluid between a rigid spherical indenter and a linear biphasic thin layer representing the articular cartilage. They indicated that this so called apparent viscosity has very small effect on the interstitial fluid flow in the cartilage layer and the matrix deformation, except near the permeable interface where a boundary layer exists.
Using the Darcy model [12] which neglects the effect of solid boundary as well as the fluid-porous media interface on fluid flow through porous media, many investigations were proposed. Typical studies are observed for non Newtonian fluid as the squeeze film rectangular porous parallel plates by Bujurke et al. [13-15] and by Naduvinamani et al. [16], the squeeze film porous spherical bearing approximating human joints by Sinha et al. [17], by Jurczak [18] and by Zaheeruddin et al. [19], the squeeze film for porous journal bearing by Zahheeruddin [20] and by Naduvinamani et al. [21,22]. For Newtonian fluid, the squeeze flow for porous circular discs was studied analytically by Murti $[23,24]$ and numerically and experimentally by Megat et al. [25]. Srinivasan [26] studied the squeeze film of Newtonian fluid between double layered annular, circular, elliptic and rectangular porous plates. It's noted that all these studies were confined to the Darcy model and thus neglected the viscous shear stresses which form a boundary layer region in the porous medium near the fluid interface. Note that the experimental results of rectilinear flow in superposed fluid and porous medium presented by Beavers and Joseph [27] have shown that the effect of the viscous shearing stresses in the fluid layer might penetrate into the permeable layer, and pointed out a slip of tangential fluid velocity on the porous interface. However, this slip flow model is only valid for a dense porous medium of large thickness. 


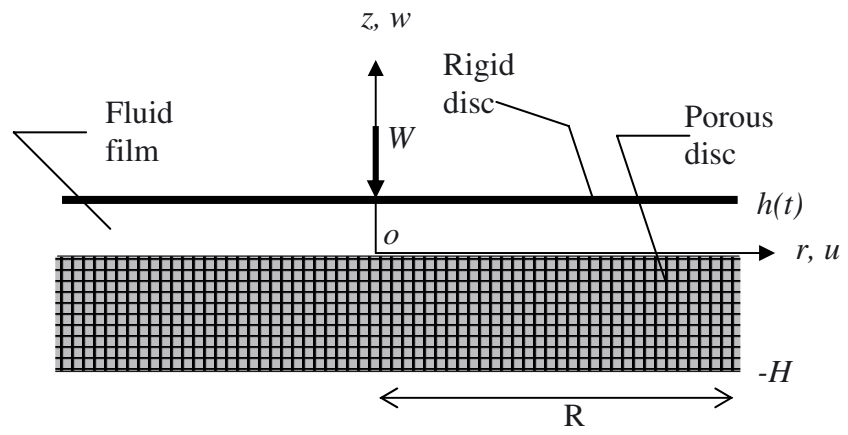

Fig. 1. Squeeze film configuration.

The Darcy-Brinkman model [28], which includes a viscous shear term, is fully compatible with the existence of a boundary layer region within the porous medium near the fluid interface. Although the boundary layer thickness is often very small, its effects on external flows can become surprisingly significant in lubrication applications. Using the Darcy-Brinkman model for lubrication applications, Lin et al. [29], Lin [30], Chen et al. [31] and Elsharkawy et al. [32] studied the lubrication performance of porous journal bearing. However, they simply considered the porous medium as a thin porous film. To examine the viscous shear effects on the squeeze film characteristics for porous circular discs, Lin [33] introduced the Darcy-Brinkman model in a simple form. Indeed, this author used the thin layer assumptions to simplify the radial component of the Darcy-Brinkman equation. On the other side, the axial component of this equation is reduced to an equation where the axial pressure gradient has a parabolic form according to the radial coordinate. So, a study accounting for more correctly the viscous shear stresses within the porous medium is needed.

The main objective of the present paper is to develop a numerical study of the viscous shear effects on the porous squeeze-film characteristics through the consideration of the complete Darcy-Brinkman equation describing the flow in porous media. The problem of two parallel circular discs when one has a porous facing is considered. Using the finite difference method, the numerical solution for the set of coupled flow governing equations is obtained iteratively. Squeeze film characteristics are then presented for various porous media. The numerical solution of the Darcy model is also included. The present numerical simulation can correct and improve the previous studies based on thin porous film assumptions.

\section{Numerical model}

We consider the porous squeeze film problem consisting of a rigid impermeable disc and a rigid porous disc separated by an incompressible lubricant of a constant viscosity as shown in Figure 1. The system build up by these two discs has an axial symmetry. The porous disc is stationary while the rigid one, supporting a constant load $W$, moves with a normal velocity $\mathrm{d} h / \mathrm{d} t$, where $h$ is the fluid film thickness. Assuming that the fluid film inertia forces are neglected and making the usual assumptions of fluid film hydrodynamic lubrication [34], the NavierStokes equations in cylindrical coordinates become:

$$
\frac{\mathrm{d} p}{\mathrm{~d} r}=\mu \frac{\partial^{2} u}{\partial z^{2}}
$$

$p, u$ and $\mu$ are respectively the pressure, the radial velocity and the dynamic viscosity of the fluid film.

The continuity equation can be written as:

$$
\frac{1}{r} \frac{\partial(r u)}{\partial r}+\frac{\partial w}{\partial z}=0
$$

where $w$ is the axial velocity. Integration of Equation (1) twice with respect to $z$ leads to the radial velocity distribution using the continuity condition $u=u^{*}$ at $z=0$ and the no slip boundary condition $u=0$ at $z=h(t)$ as:

$$
u(r, z)=\frac{1}{2 \mu} \frac{\mathrm{d} p}{\mathrm{~d} r}\left(z^{2}-z h\right)+u_{0}^{*}\left(1-\frac{z}{h}\right)
$$

By replacing into the continuity equation (2) the velocity component $u=u(r, z)$ with its expression from Equation (3) and integrating with respect to $z$, using the continuity condition $w=w^{*}$ at $z=0$ and the no slip boundary condition $w=\mathrm{d} h / \mathrm{d} t$ at $z=h(t)$, the modified Reynolds equation can be derived as:

$$
\frac{1}{r} \frac{\mathrm{d}}{\mathrm{d} r}\left(r \frac{\mathrm{d} p}{\mathrm{~d} r}\right)=\frac{12 \mu}{h^{3}}\left(\frac{\mathrm{d} h}{\mathrm{~d} t}+\frac{h}{2 r} \frac{\mathrm{d}\left(r u_{0}^{*}\right)}{\mathrm{d} r}-w_{0}^{*}\right)
$$

where $u_{0}^{*}$ and $w_{0}^{*}$ denote the radial and the axial velocities of the fluid at the porous disc-film interface.

The porous disc is considered isotropic and of a constant permeability. In this study, the inertia effects can be neglected as the Reynolds number $R_{\mathrm{ep}}=-\frac{\rho \sqrt{k} \mathrm{~d} h / \mathrm{d} t}{\mu}[35]$ based on the porous medium permeability $k$ is small and its values are less than 0.01 , here $\rho$ is the fluid density. Thus, the flow in this medium is described by the DarcyBrinkman equation [28] which is usually used to describe the low Reynolds number flow in highly porous media (with porosity greater than 0.6) [36]:

$$
\begin{aligned}
u^{*} & =-\frac{k}{\mu} \frac{\partial p^{*}}{\partial r}+k \frac{\mu^{*}}{\mu}\left(\frac{\partial}{\partial r}\left(\frac{1}{r} \frac{\partial\left(r u^{*}\right)}{\partial r}\right)+\frac{\partial^{2} u^{*}}{\partial z^{2}}\right) \\
w^{*} & =-\frac{k}{\mu} \frac{\partial p^{*}}{\partial z}+k \frac{\mu^{*}}{\mu}\left(\frac{1}{r} \frac{\partial}{\partial r}\left(r \frac{\partial w^{*}}{\partial r}\right)+\frac{\partial^{2} w^{*}}{\partial z^{2}}\right)
\end{aligned}
$$

where $p^{*}$ is the pressure, $\mu^{*}$ is the fluid effective viscosity which is different from the fluid viscosity $\mu, u^{*}$ and $w^{*}$ are the radial and the axial fluid velocities in the porous disc. For the simplest cases, i.e. isotropic porous media made of regular-shaped and narrowly size-distributed solid grains, the value of the permeability $k$ can be approximated using the phenomenological Carman-Kozeny formula [36]: $k=D_{\mathrm{p}}^{2} \phi^{3} / 180(1-\phi)^{2}$, where $D_{\mathrm{p}}$ is the characteristic pore diameter and $\phi$ is the porosity. In this study, $\phi$ does not take place explicitly in our equations. 
The right hand side terms in Equations (5) and (6) denote namely the Darcy term and the Brinkman term respectively. The viscous shear effects are characterised by the dimensionless parameter $\alpha=\mu^{*} / \mu$. The effective viscosity is known to be strongly dependent on the porosity as well as the geometrical structure of the porous media [36], but is not experimentally documented and is still subject to investigation. Liu and Masliyah [37] summarize the present understanding saying that the numerical simulations have shown that, depending upon the type of porous media, the effective viscosity may be either smaller or greater than the viscosity of the fluid. By means of an up-scaling procedure, based on the volume averaging methods of the Stokes equations of the microscopic flow through porous media, Valdes-Parada et al. [38] pointed out from their results that the effective viscosity should be a decreasing function of the porous medium porosity. Initially, the effective viscosity was assumed by Brinkman to be equal to the fluid viscosity [36], but the process of the local volumetric averaging of the microscopic flow through the pores leads to the relationship [39]: $\mu^{*}=\mu / \phi$.

Taking the divergence of the velocity vector given by Equations (5), (6) and considering the flow to be incompressible in the porous disc, the continuity equation can be replaced by the Laplace equation:

$$
\frac{1}{r} \frac{\partial}{\partial r}\left(r \frac{\partial p^{*}}{\partial r}\right)+\frac{\partial^{2} p^{*}}{\partial z^{2}}=0
$$

The computational domain is reduced to $0 \leq r \leq R$ and $-H \leq z \leq h(t)$ for geometry symmetry reasons. The boundary conditions associated to the system of Equations (1), (2), (4), (5), (6) and (7) are:

- on the porous disc - fluid film interface $z=0$ :

$$
u=u^{*}, w=w^{*}, p=p^{*}, \mu \frac{\partial u}{\partial z}=\mu^{*} \frac{\partial u^{*}}{\partial z}, \frac{\partial w^{*}}{\partial z}=0
$$

- on the upper disc $z=h(t)$ :

$$
u=0, w=\frac{\mathrm{d} h}{\mathrm{~d} t}
$$

- on the symmetry axis $r=0$ :

$$
u=u^{*}=0, \frac{\partial w}{\partial r}=\frac{\partial w^{*}}{\partial r}=0, \frac{\mathrm{d} p}{\mathrm{~d} r}=\frac{\partial p^{*}}{\partial r}=0
$$

- on the lower wall $z=-H$ :

$$
u^{*}=w^{*}=0, \frac{\partial p^{*}}{\partial z}=0
$$

- on the edge of porous disc - fluid film domain $r=R$ :

$$
p=p^{*}=0, \frac{\partial u^{*}}{\partial r}=\frac{\partial w^{*}}{\partial r}=0
$$

where conditions in (8) imply the continuity of the fluid velocity, of the pressure, of the normal stress and of the tangential stress at the porous interface. This later condition is obtained from the balance of the normal components of the fluid stress across the interface when the pressure is assumed to be continuous across the interface and the term of normal viscous stress is negligible. Conditions in (9) are the no-slip conditions, conditions (10) are due to symmetry, conditions (11) are the no-slip and the non permeability conditions respectively, conditions (12) are respectively the zero ambient pressure and the "continuative" condition consisting of a zero normal derivatives for the flow field velocity, which is intended to represent a smooth continuation of the flow through the edge of the porous disc.

Introducing following non-dimensional parameters and variables:

$\bar{r}=\frac{r}{R}, \bar{z}=\frac{z}{h}$ when $\bar{z}>0$ and $\bar{z}=\frac{z}{H}$ when $\bar{z}<0, \bar{u}=-\frac{h}{R \mathrm{~d} h / \mathrm{d} t} u, \bar{w}=-\frac{w}{\mathrm{~d} h / \mathrm{d} t}, \bar{p}=-\frac{p h^{3}}{\mu R^{2} \mathrm{~d} h / \mathrm{d} t}$, $\bar{u}^{*}=-\frac{h}{R \mathrm{~d} h / \mathrm{d} t} u^{*}, \bar{w}^{*}=-\frac{w^{*}}{\mathrm{~d} h / \mathrm{d} t}, \bar{p}^{*}=-\frac{p^{*} h^{3}}{\mu R^{2} \mathrm{~d} h / \mathrm{d} t}$, Equations (1), (2), (4), (5), (6) and (7) become:

$$
\begin{gathered}
\frac{\mathrm{d} \bar{p}}{\mathrm{~d} \bar{r}}=\frac{\partial^{2} \bar{u}}{\partial \bar{z}^{2}} \\
\frac{1}{\bar{r}} \frac{\partial(\overline{r u})}{\partial \bar{r}}+\frac{\partial \bar{w}}{\partial \bar{z}}=0 \\
\frac{1}{\bar{r}} \frac{\mathrm{d}}{\mathrm{d} \bar{r}\left(\bar{r} \frac{\mathrm{d} \bar{p}}{\mathrm{~d} \bar{r}}\right)=} 12\left(-1+\frac{1}{2 \bar{r}} \frac{\mathrm{d}\left(\bar{r} \bar{u}_{0}^{*}\right)}{\mathrm{d} \bar{r}}-\bar{w}_{0}^{*}\right) \\
\bar{u}^{*}=-\frac{k}{h^{2}} \frac{\partial \bar{p}}{\partial \bar{r}}+\frac{k}{H^{2}} \frac{\mu^{*}}{\mu}\left(\left(\frac{H}{R}\right)^{2} \frac{\partial}{\partial \bar{r}}\left(\frac{1}{\bar{r}} \frac{\partial\left(\overline{r u}^{*}\right)}{\partial \bar{r}}\right)+\frac{\partial^{2} \bar{u}^{*}}{\partial \bar{z}^{2}}\right) \\
\bar{w}^{*}=-\frac{k R^{2}}{H h^{3}} \frac{\partial \bar{p}^{*}}{\partial \bar{z}}+\frac{k}{H^{2}} \frac{\mu^{*}}{\mu}\left(\left(\frac{H}{R}\right)^{2} \frac{1}{\bar{r}} \frac{\partial}{\partial \bar{r}}\left(16 \frac{\partial \bar{w}^{*}}{\partial \bar{r}}\right)+\frac{\partial^{2} \bar{w}^{*}}{\partial \bar{z}^{2}}\right) \\
\left(\frac{H}{R}\right)^{2} \frac{1}{\bar{r}} \frac{\partial}{\partial \bar{r}}\left(\bar{r} \frac{\partial \bar{p}^{*}}{\partial \bar{r}}\right)+\frac{\partial^{2} \bar{p}^{*}}{\partial \bar{z}^{2}}=0
\end{gathered}
$$

When using dimensionless form we observe the disappearance of the unknown squeeze velocity $\mathrm{d} h / \mathrm{d} t$ which is calculated later using the load capacity relationship.

The dimensionless boundary conditions are:

- on the porous disc - fluid film interface $\bar{z}=0$ :

$$
\bar{u}=\bar{u}^{*}, \bar{w}=\bar{w}^{*}, \bar{p}=\bar{p}^{*}, \frac{\partial \bar{u}}{\partial \bar{z}}=\frac{\mu^{*}}{\mu} \frac{h}{H} \frac{\partial \bar{u}^{*}}{\partial \bar{z}}, \frac{\partial \bar{w}^{*}}{\partial \bar{z}}=0
$$

- on the upper disc $\bar{z}=1$ :

$$
\bar{u}=0, \bar{w}=-1
$$

- on the symmetry axis $\bar{r}=0$ :

$$
\bar{u}=\bar{u}^{*}=0, \frac{\partial \bar{w}}{\partial \bar{r}}=\frac{\partial \bar{w}^{*}}{\partial \bar{r}}=0, \frac{\mathrm{d} \bar{p}}{\mathrm{~d} \bar{r}}=\frac{\partial \bar{p}^{*}}{\partial \bar{r}}=0
$$

- on the lower wall $\bar{z}=-1$ :

$$
\bar{u}^{*}=\bar{w}^{*}=0, \frac{\partial \bar{p}^{*}}{\partial \bar{z}}=0
$$




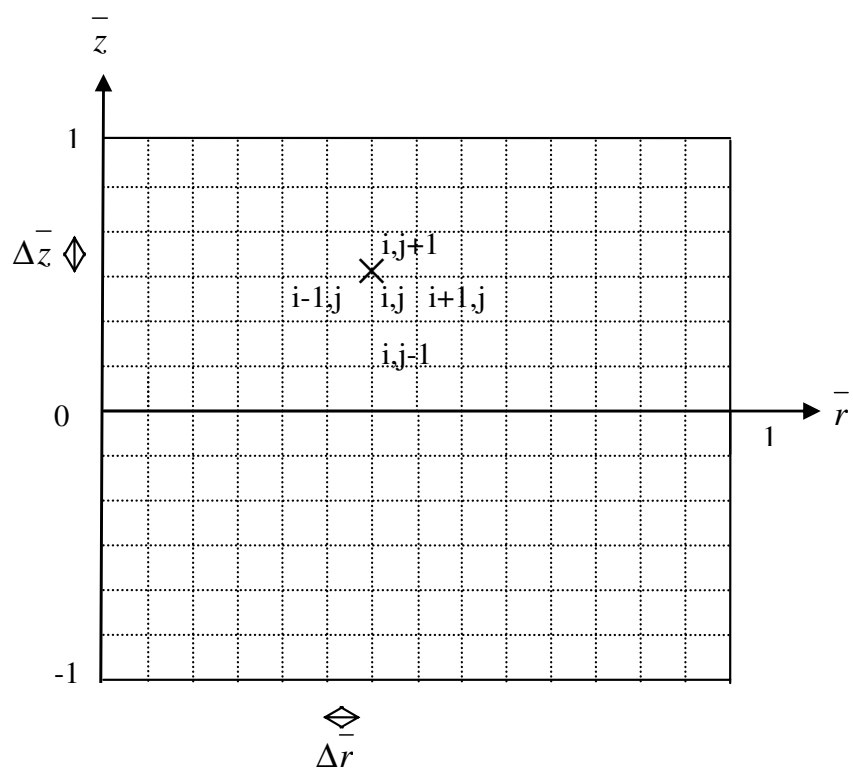

Fig. 2. Film and porous medium discretisation.

- on the edge of porous disc - fluid film domain $\bar{r}=1$ :

$$
\bar{p}=\bar{p}^{*}=0, \frac{\partial \bar{u}^{*}}{\partial \bar{r}}=\frac{\partial \bar{w}^{*}}{\partial \bar{r}}=0
$$

The coupled dimensionless differential equations subjected to the above dimensionless boundary conditions are discretized using the finite difference method. Constant grid sizes were implemented in the $\bar{r}$ and $\bar{z}$ directions (see Fig. 2). Central differentiation was used for the spatial derivatives except for the axial velocity derivative in the continuity equation, for which the first order backward scheme was employed. The resulting set of algebraic equations was solved using the successive over relaxation scheme (SOR). A $51 \times 51$ grid configuration was found to satisfy the grid independence results by a systematic decrease in the grid size for the film and the porous medium. The scheme was assumed to have converged when the maximum root mean square change for the variables $\bar{u}$, $\bar{w}, \bar{p}, \bar{u}^{*}, \bar{w}^{*}, \bar{p}^{*}$ between two consecutive iterations is below the convergence criterion of $10^{-9}$. In what follows, a brief description of the procedure for the numerical solution is provided. First, starting from an initial fluid film thickness $h_{0}$, the dimensionless hydrodynamic variables in the film and in the porous medium are set to zero. Using an iterative scheme, the solution of the dimensionless Equations (13)-(17) and (18) is achieved when the convergence criterion is satisfied. Since the dimensionless film pressure profile is calculated, the dimensionless load carrying capacity $\bar{W}$ can be determined. So, the squeeze film velocity is deduced from the relationship:

$$
-\mathrm{d} h / \mathrm{d} t=\frac{W h^{3}}{\mu R^{4} \bar{W}}
$$

This solution procedure is repeated, by decreasing the film thickness and estimating new values of $\bar{u}, \bar{w}, \bar{p}, \bar{u}^{*}, \bar{w}^{*}$ and $\bar{p}^{*}$, until the fluid film thickness is equal to its minimum
Table 1. Physical and geometrical data.

\begin{tabular}{lc}
\hline Fluid density (kg.m ${ }^{-3}$ ) & 840 \\
Dynamic viscosity (Pa.s) at $20{ }^{\circ} \mathrm{C}$ & 0.086 \\
Porous disc permeability $\left(\mathrm{m}^{2}\right)$ & $4.73 \times 10^{-11}$ \\
Porous disc thickness (mm) & 1.6 \\
Discs radius (mm) & 40 \\
Initial fluid film thickness (mm) & 1.2 \\
Applied load (N) & 87 \\
\hline
\end{tabular}

value. Finally, the response time of the squeeze film is calculated by numerical integration of Equation (24) with respect to $h$. This sequence is shown as a flow chart in Figure 3.

\section{Results and discussions}

In the present study, the geometrical and physical parameters are the same as in Megat et al. [25] (see Tab. 1). As we use in this investigation the same fluid, the value of the parameter $\alpha\left(=\mu^{*} / \mu\right)$, providing the viscous shear effects of the Darcy-Brinkman model, depends upon the variation of the porosity. To illustrate the effects on the viscous shear effects and the variation of $\alpha$ on the squeeze film performances, the results for different porous media predicted by the present model are compared to those derived by the Darcy model. Figure 4 shows the pressure distribution in the fluid film as a function of the radius. In the case of the Darcy-Brinkman model, it is found that the viscous shear stresses effects on the fluid film pressure are not significant for three different considered porous media with $\alpha=1,2$ and 4 . From the graph it can be observed that the curves shapes are the same as those obtained when using the Darcy model, showing a radial decay in pressure. This result is in good agreement with that obtained by Megat et al. [25], when the Darcy model is used over a central part of the bearing. This profile form of the pressure is in agreement with the result from Murti [23], and was stated by Megat et al. [25]. Figure 5 illustrates the pressure distribution within the porous medium, for example when $\alpha=4$. It can be seen that the variation of the pressure against the axial component is small in comparison with its variation with respect to the radial component. The fluid flow is essentially in the radial direction. Moreover, the viscous shear effects have no significant effects on the pressure for all considered values of $\alpha$.

The effects of the viscous shearing forces on the fluid velocity profiles are depicted in Figures 6 and 7 at the fluid film thicknesses $h=1 \mathrm{~mm}$ (case a) and $h=0.2 \mathrm{~mm}$ (case b). From Figure 6, we observe that the radial velocity has a nearly parabolic shape in fluid film but it is almost constant across the porous disc, except in the immediate vicinity of the fluid interface where the boundary layer effects take clearly place. The viscous shear stresses have no significant effects on the radial velocity in the porous medium, except obviously in the boundary layer region near the fluid interface where it becomes 


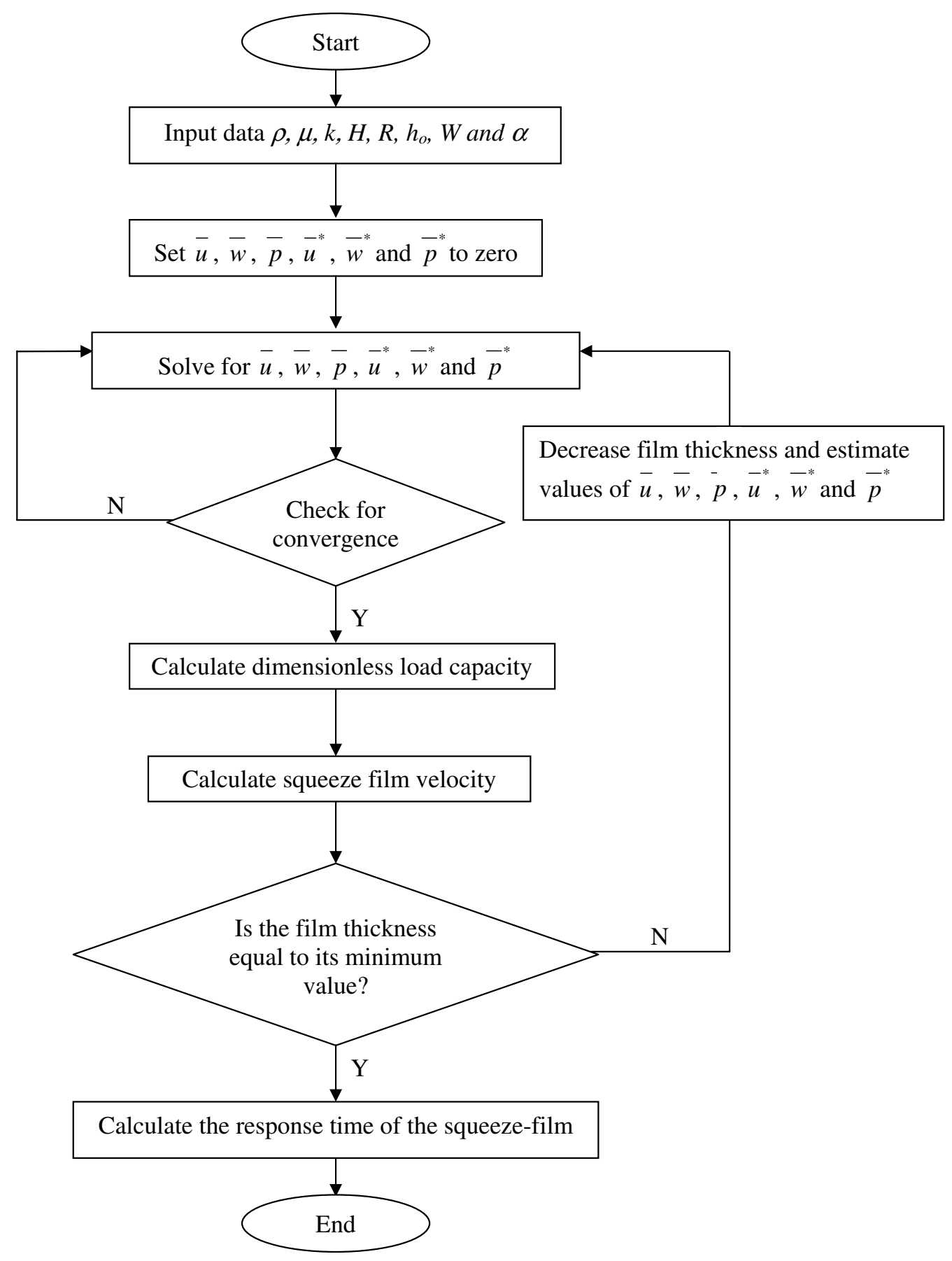

Fig. 3. Computer program flow chart.

easier for the fluid to flow radially when using the DarcyBrinkman model. As a result, the minimum values of the radial velocity in the fluid film are obtained for the Darcy model since the viscous shearing stresses allow the fluid flow to be more rapid at the fluid interface. In addition, the fluid flows more rapidly, in the film and the boundary layer within the porous medium. This is observed when decreasing the parameter $\alpha$, i.e. when reducing the resistance of the viscous forces to the fluid flow. Moreover, the location of maximum radial velocity shifts slightly towards the fluid film - porous disc interface when decreasing $\alpha$. These effects are more pronounced when the film thickness separating the two discs becomes smaller.

The axial velocity, Figure 7, decreases almost linearly in the film and more significantly in the porous medium. The viscous shear forces have no significant effects on the axial velocity across the porous medium as they are missing in the fluid film especially at the permeable interface. However, it is observed that the Darcy model underestimates the values of this velocity in the fluid film essentially at the upper disc. Since the viscous shear stresses result in a higher radial flow rate across the film edge 


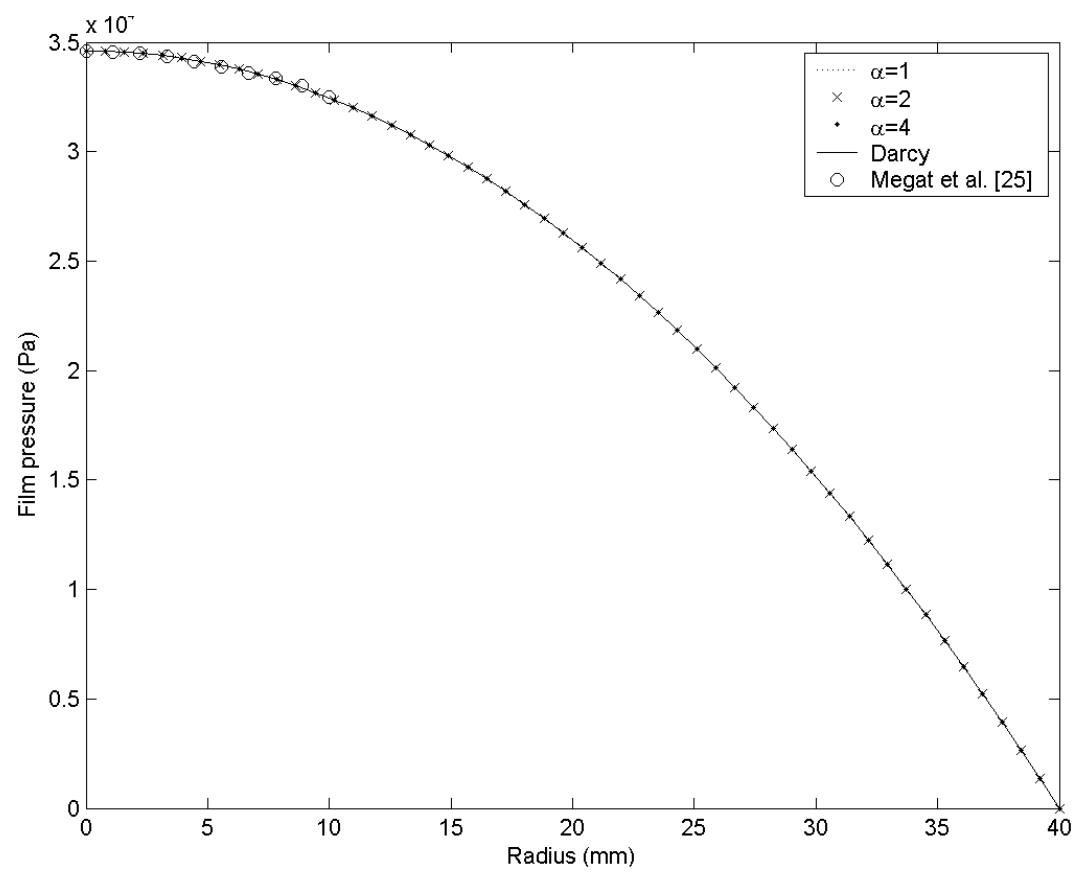

Fig. 4. Pressure distribution in the fluid film.

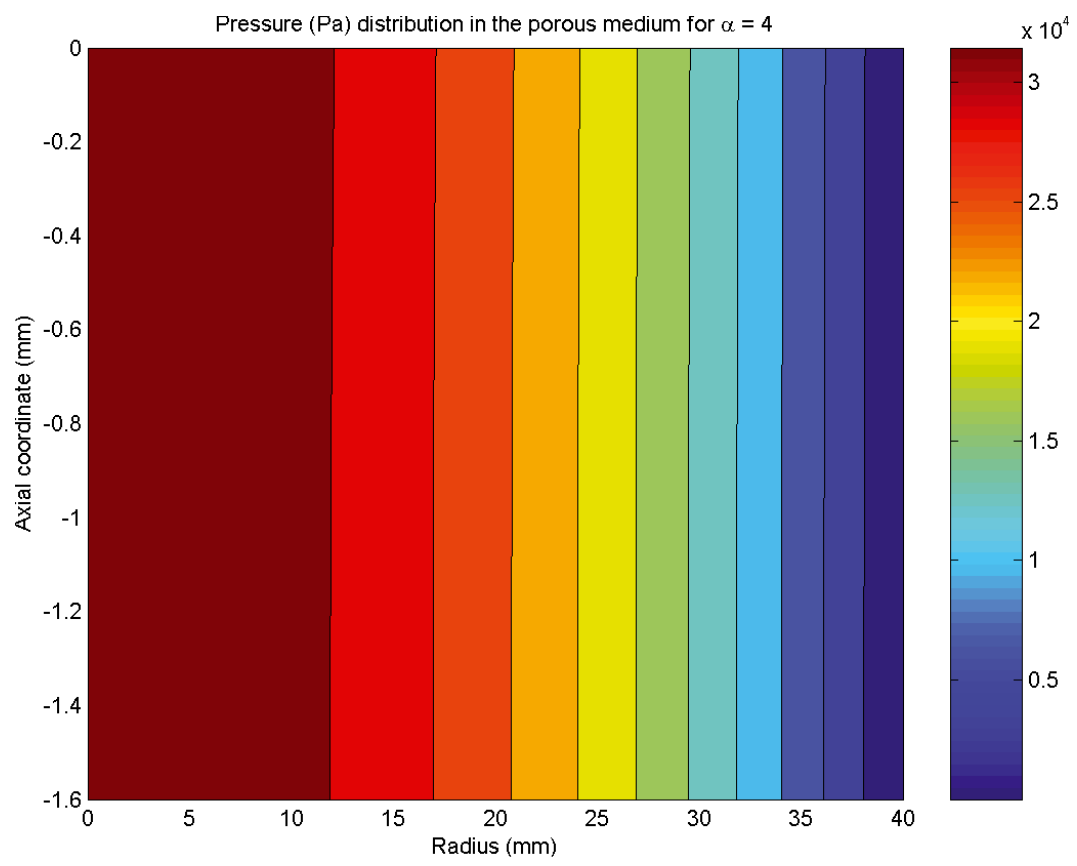

Fig. 5. Pressure distribution in the porous medium.

when comparing with the Darcy model, the upper disc will move more fastly. On the other hand, decreasing $\alpha$ leads to an increase in the axial velocity between the two discs. It's also interesting to note that the effect of the parameter $\alpha$ and of its variations on the axial fluid film velocity is enlarged as the fluid film thickness decreases.

Figure 8 displays the comparison of the fluid film thickness evolution with time predicted by the Darcy model and the Darcy-Brinkman model for three different porous media. It's noted that the response time required for the upper disc to attain a given film height $h$ increases when increasing the effective viscosity. Decreasing the parameter $\alpha$ reduces the resistance against the radial fluid flow in the porous media, and as a result a lower value of $h$ is reached for the same time. Moreover, these effects become increasingly large when the rigid disc continues to approach the porous disc. When comparing with the Darcy model, the viscous shear effects of the 


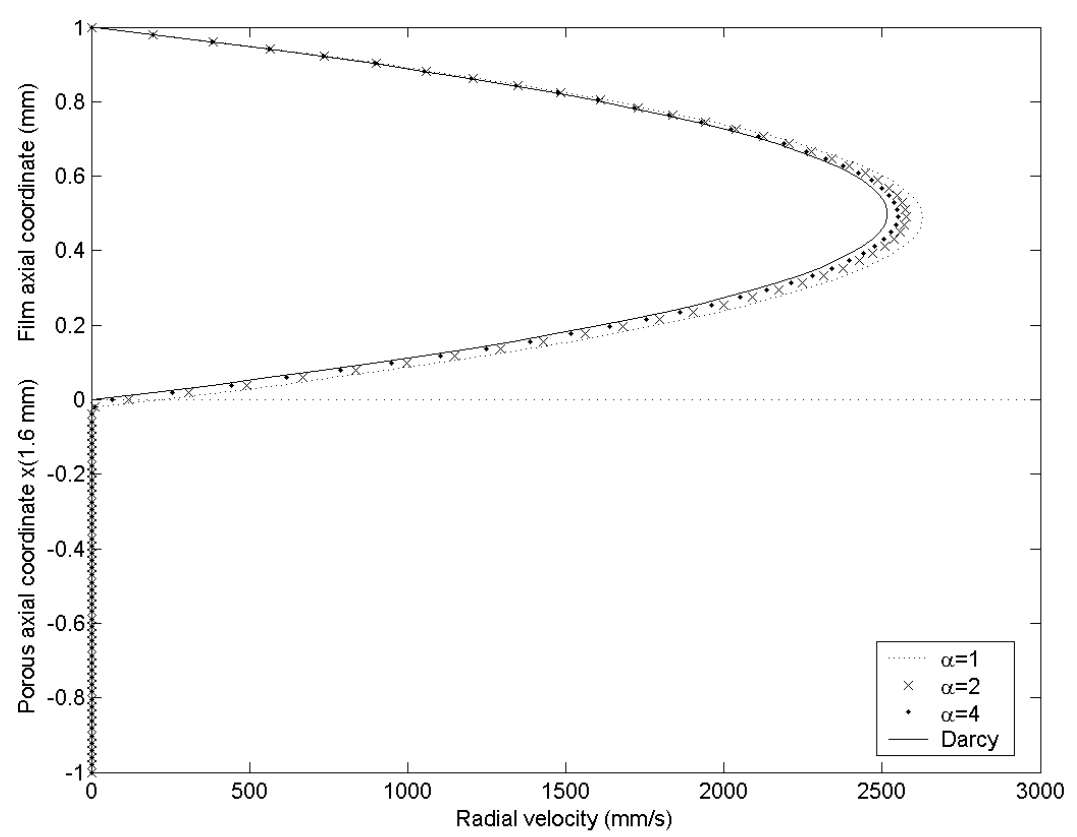

Case a: $h=1 \mathrm{~mm}$.

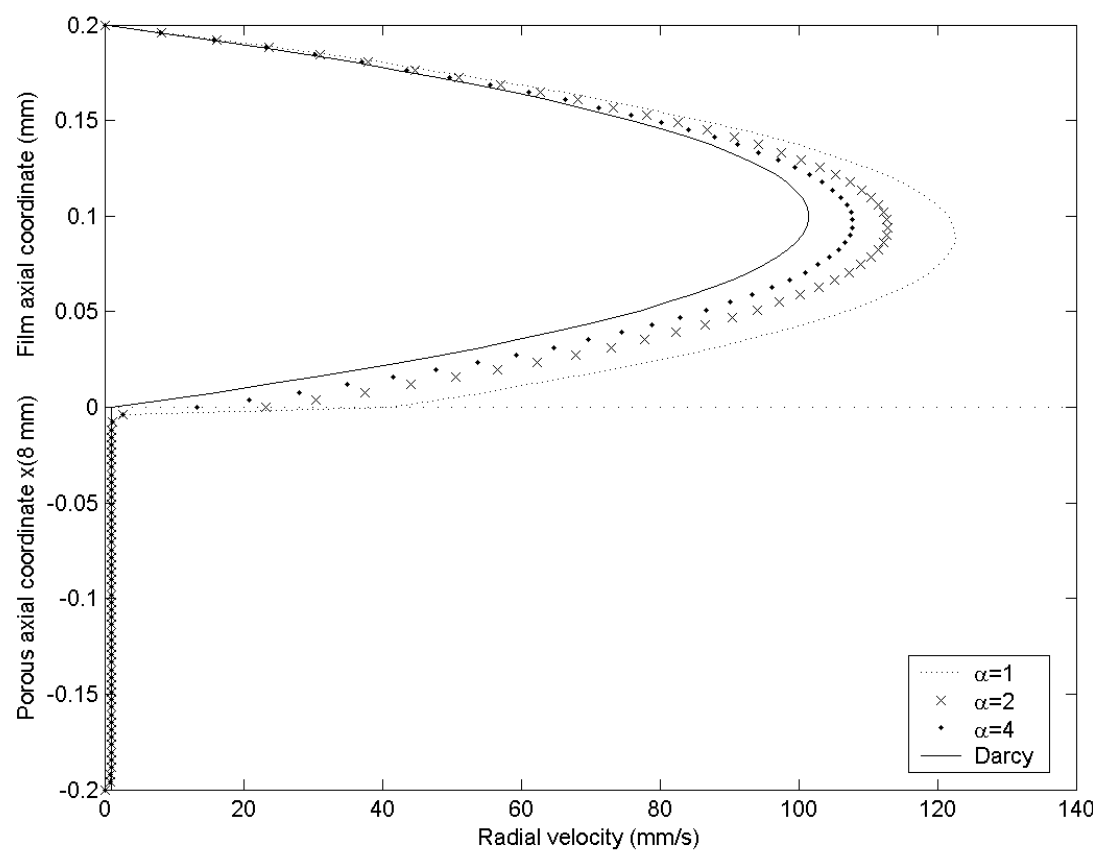

Case b: $h=0.2 \mathrm{~mm}$.

Fig. 6. Radial velocity profiles at $r=R$ for $h=1 \mathrm{~mm}$ and $h=0.2 \mathrm{~mm}$.

Darcy-Brinkman model reduce the response time of the squeeze-film. Since the Darcy-Brinkman model results in a higher flow rate across the film edge when compared to the Darcy model, a lower value of $h$ is reached for the same response time to be taken. This is in excellent agreement with the results from Lin [33].

Results on the squeeze velocity evolution $\mathrm{d} h / \mathrm{d} t$ are shown in Figure 9. It can be observed that, when comparing the Darcy's model and the Darcy-Brinkman model, accounting for the viscous shear stresses leads to higher squeeze velocities. The viscous shear stresses reduce the resistance encountered by the radial flow at the porous interface and thus the fluid can be squeezed out more easily, which allows the upper disc to move more quickly.

By contrast with the results of Lin [30,33], our finding pointed out that the effects of the variation of the 


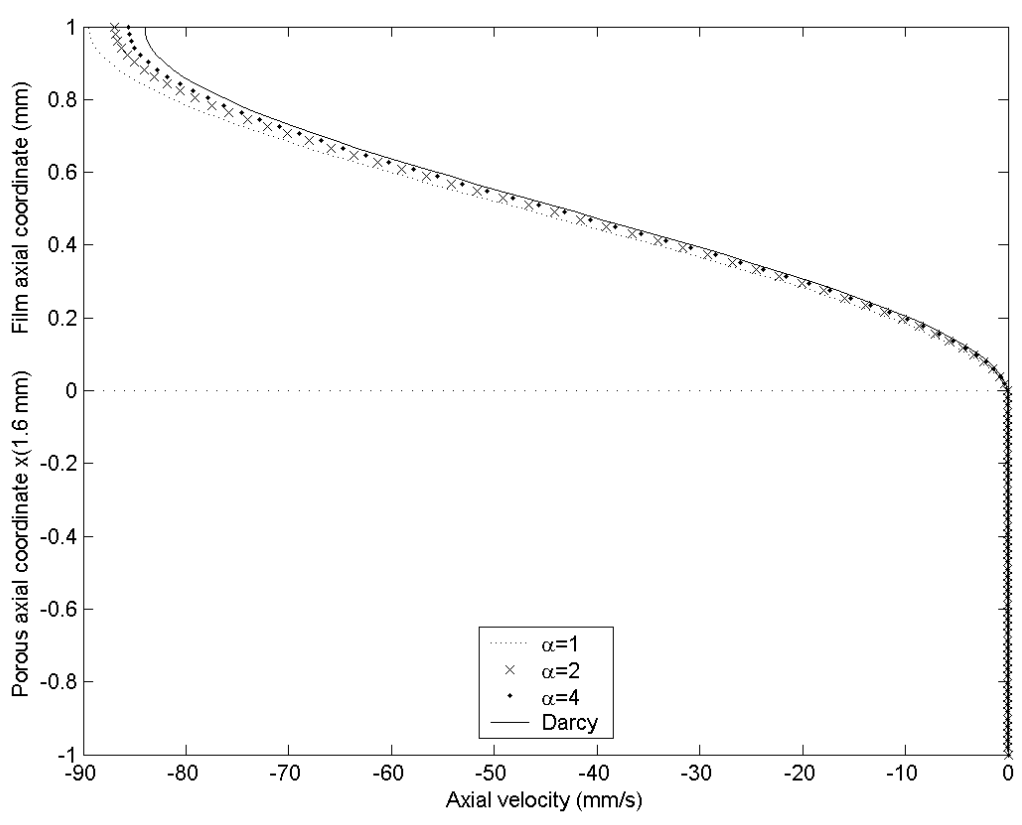

Case a: $h=1 \mathrm{~mm}$.

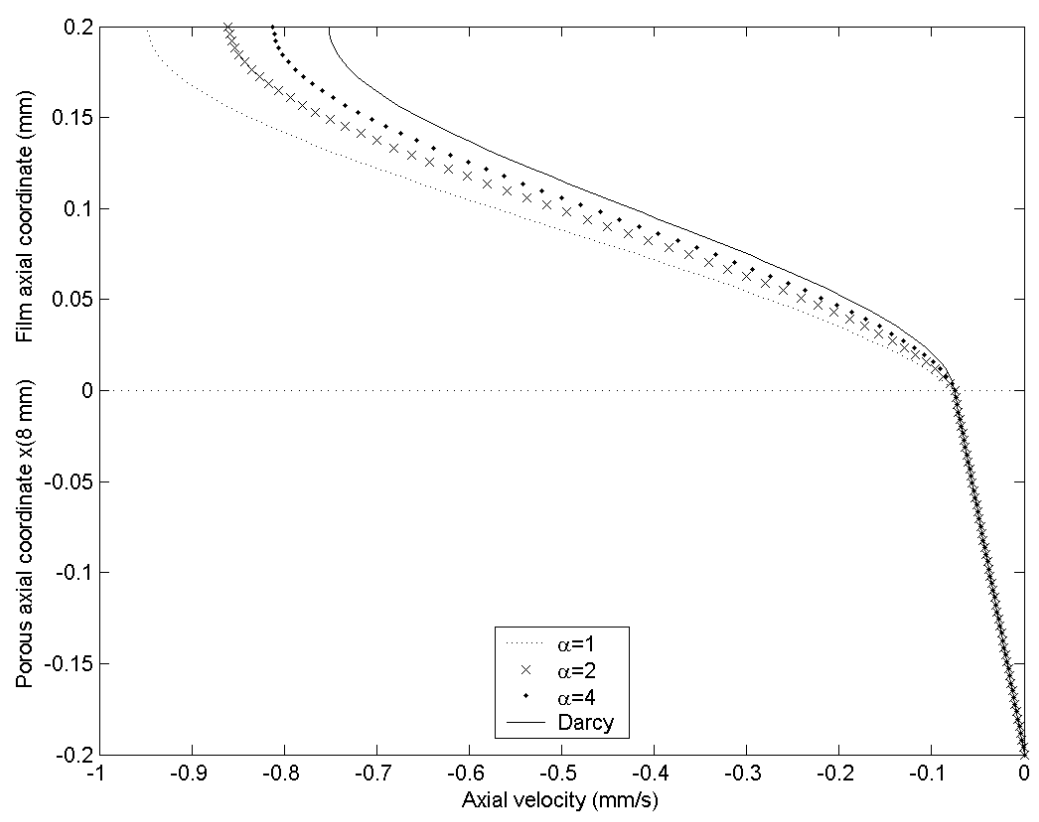

Case b: $h=0.2 \mathrm{~mm}$.

Fig. 7. Axial velocity profiles at $r=0$ for $h=1 \mathrm{~mm}$ and $h=0.2 \mathrm{~mm}$.

viscosity ratio $\alpha$ on the squeeze film characteristics are significant and thus not negligible.

\section{Conclusion}

A numerical investigation of viscous shear effects on the squeeze-film characteristics has been presented using the modified Reynolds equation and the Darcy-Brinkman equations. Considering the Darcy-Brinkman equation which is usually used to describe the low Reynolds number flow in highly porous media is intimately linked to squeezing situations where the porous medium has a porosity greater than 0.6 . It's found that the parameter $\alpha$ of shear viscous shearing and its variation show significant and not negligible effects of the viscous shearing stresses on the squeeze-film characteristics, essentially for low values of $\alpha$. As the parameter $\alpha$ increases (the porosity decreases), the results predicted by using the DarcyBrinkman model approach the Darcy model solution. The 


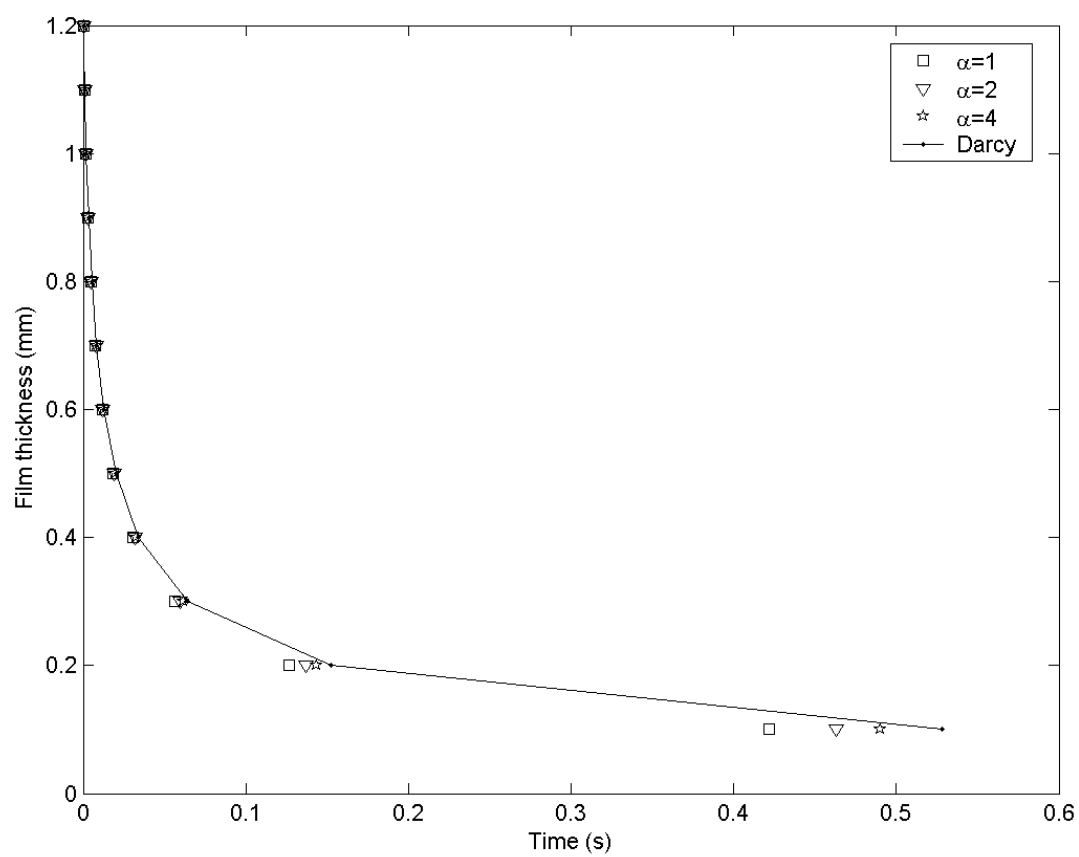

Fig. 8. Film thickness.

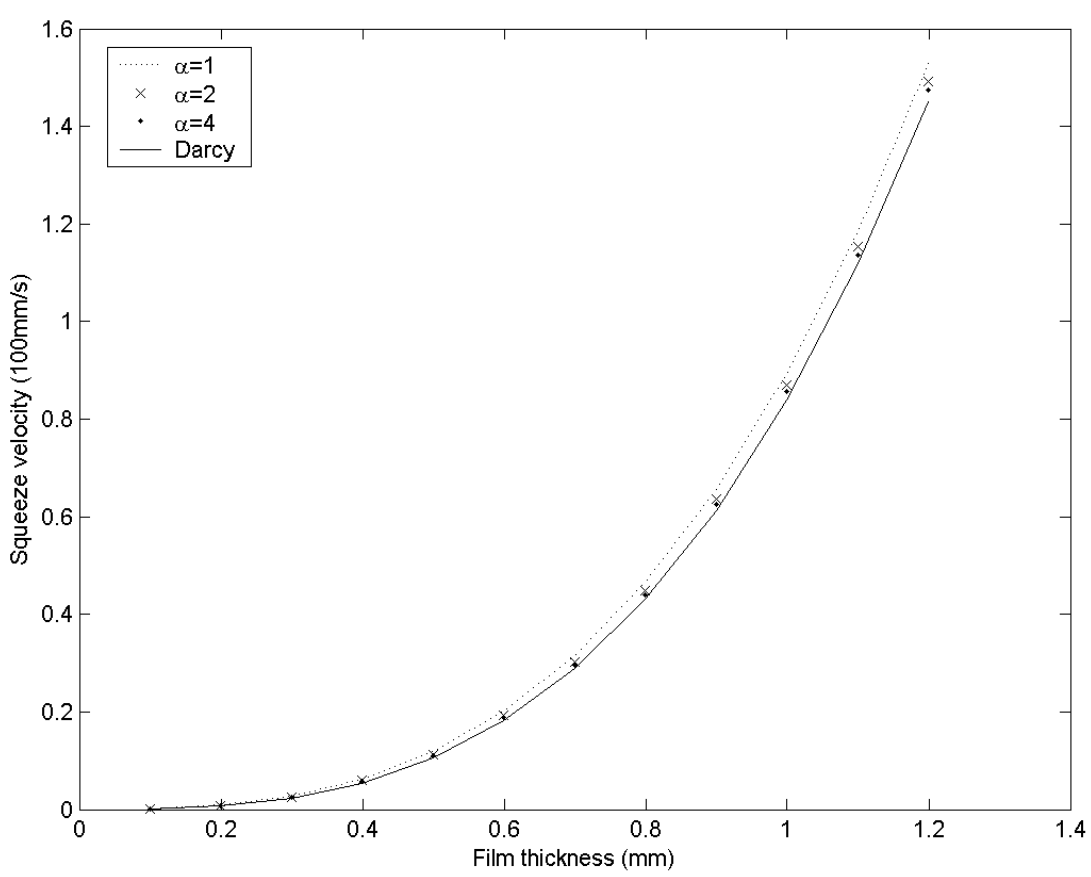

Fig. 9. Squeeze film velocity versus the film thickness.

viscous shear effects of the Darcy-Brinkman model reduce the response time of the squeeze-film but increase the squeeze film velocity, the radial and the axial velocities, compared to the Darcy model. Moreover, these effects are more pronounced as the fluid film thickness decreases. Nevertheless there are some restrictions on the validity of the Darcy-Brinkman equation to model high porosity media mainly the non-slip condition at the bounding solid walls [40].
Acknowledgements. The authors would like to thank the French-Moroccan Mixed Inter-University Committee for supporting this work under Grant number SPI 06/10.

\section{References}

[1] N.M. Bujurke, H.P. Patil, An analysis of squeeze film action in porous layered bearings, Wear 145 (1991) 385-397 
[2] N.M. Bujurke, R.B. Kudenatti, Surface roughness effects on squeeze film poroelastic bearings, Appl. Math. Comput. 174 (2006) 1181-1195

[3] N.M. Bujurke, C.S. Salimath, R.B. Kudenatti, S.C. Shiralashetti, Wavelet-multigrid analysis of squeeze film characteristics of poroelastic bearings, J. Comput. Appl. Math. 203 (2007) 237-248

[4] N.M. Bujurke, R.B. Kudenatti, V.B. Awati, Effects of roughness on squeeze film poroelastic bearings with special reference to synovial joints, Math. Biosc. 209 (2007) 76-89

[5] P.N. Tandon, J.K. Misra, R.S. Gupta, Satyanand, Role of ultrafiltration of synovial fluid in lubrication of human joints, Int. J. Mech. Sci. 27 (1985) 29-37

[6] M. Hlavacek, The role of synovial fluid filtration by cartilage in lubrication of synovial joints - II, Squeeze-film lubrication: homogeneous filtration, J. Biomech. 26 (1993) $1151-1160$

[7] M. Hlavacek, The role of synovial fluid filtration by cartilage in lubrication of synovial joints - III, Squeeze-film lubrication: axial symmetry under low loading conditions, J. Biomech. 28 (1995) 1193-1195

[8] M. Hlavacek, The role of synovial fluid filtration by cartilage in lubrication of synovial joints - IV, Squeeze-film lubrication: the central film thickness for normal and inflammatory synovial fluids for axial symmetry under high loading conditions, J. Biomech. 28 (1995) 1199-1205

[9] M. Hlavacek, Squeeze-film lubrication of the human ankle joint with synovial fluid filtrated by articular cartilage with the superficial zone worn out, J. Biomech. 33 (2000) $1415-1422$

[10] M. Hlavacek, The influence of the acetabular labrum seal intact articular superficial zone and synovial fluid thixotropy on squeeze-film lubrication of spherical synovial joint, J. Biomech. 35 (2002) 1325-1335

[11] J.S. Hou, V.C. Mow, W.M. Lai, M.H. Holmes, An analysis of the squeeze-film lubrication mechanics for articular cartilage, J. Biomech. 25 (1992) 247-259

[12] A.E. Scheidigger, The physics of flow through porous media, The MacMillan Co., New York, 1957

[13] N.M. Bujurke, M. Jagadeeswar, P.S. Hiremath, Analysis of normal stress effects in a squeeze film porous bearings, Wear 116 (1987) 237-248

[14] N.M. Bujurke, M. Jagadeeswar, P.S. Hiremath, Analysis of squeeze film lubrication between two rectangular porous plates with a second-order fluid, Wear 132 (1989) 303-320

[15] N.M. Bujurke, N.B. Naduvinamani, A note on squeeze film between rough anisotropic porous rectangular plates, Wear 217 (1998) 225-230

[16] N.B. Naduvinamani, S.T. Fathima, P.S. Hiremath, Effect of surface roughness on characteristics of couple stress squeeze film between anisotropic porous rectangular plates, Fluid Dyn. Res. 32 (2003) 217-231

[17] P. Sinha, C. Singh, K.R. Prasad, Lubrication of human joints - a microcontinum approach, Wear 80 (1982) 159181

[18] P. Jurczak, Pressure distribution in a squeeze film biobearing lubrication by a synovial fluid, Int. J. Appl. Mech. Eng. 11 (2006) 857-864

[19] K.H. Zaheerduddin, M. Isa, Characteristics of a micropolar lubricant in a squeeze film porous spherical bearing, Wear 52 (1978) $1-10$
[20] K.H. Zaheerduddin, The dynamic behaviour of squeeze films in one-dimensional porous journal bearing lubricated by a micropolar fluid, Wear 71 (1981) 139-152

[21] N.B. Naduvinamani, P.S. Hiremath, G. Gurubasavaraj, Squeeze film lubrication of short porous journal bearing with couple stress fluids, Tribol. Int. 34 (2001) 739-747

[22] N.B. Naduvinamani, S.B. Patil, Numerical solution of finite modified Reynolds equation for couple stress squeeze film lubrication of porous journal bearings, Comput. Struct. 87 (2009) 1287-1295

[23] P.R.K. Murti, Squeeze films in porous circular disks, Wear 23 (1973) 283-289

[24] P.R.K. Murti, Squeeze-film behaviour in porous circular disks, ASME J. Lubr. Techno 96 (1974) 206-209

[25] M.M.H. Megat Ahmad, D.T. Gethin, T.C. Claypole, B.J. Roylance, Numerical and experimental investigation into porous squeeze films, Tribol. Int. 31 (1998) 189-199

[26] U. Srinvasan, Load capacity and time-height relationships for squeeze films between double-layered porous plates, Wear 43 (1977) 211-225

[27] G.S. Beavers, D.D. Joseph, Boundary conditions on a naturally permeable wall, J. Fluid Mech. 30 (1967) 197-207

[28] H.C. Brinkman, A calculation of the viscous force exerted by a flowing fluid on a dense swarm of particles, Appl. Sci. Res. A1 (1947) 27-34

[29] J.R. Lin, C.C. Hwang, Lubrication of short porous journal bearing - use of the Brinkman-extended Darcy model, Wear 161 (1993) 93-104

[30] J.R. Lin, Optimal design of one-dimensional porous slider bearing using the Brinkman model, Trib. Int. 34 (2001) $57-64$

[31] Ming-Da Chen, Kuo-Ming Chang, J.R. Lin, W.L. Li, Lubrication of journal bearings - influence of stress jump condition at porous media/fluid film interface, Trib. Int. 35 (2002) 287-295

[32] A. Elsharkawy, L.H. Guedouar, Hydrodynamic lubrication of porous journal bearings using a modified Brinkman-extended Darcy model, Trib. Int. 34 (2001) 767-777

[33] R.J. Lin, Viscous shear effects on the squeeze-film behaviour in porous circular disks, Int. J. Mech. Sci. 38 (1996) 373-384

[34] A. Cameron, Basic Lubrication Theory, Wiley Eastern Ltd, New York, 1987

[35] J.R.A. Pearson, P.M.J. Tardy, Models for flow of non Newtonian and complex fluids through porous media, J. Non Newtonian Fluid Mech. 102 (2002) 447-473

[36] D.A. Nield, A. Bejan, Convection in porous media 2nd edition, Spring-verlag: New York, 1999

[37] S. Liu, J.H. Masliyah, Dispersion in porous media. Handbook of Porous Media, K. Vafai (ed.), 2nd edition, Taylor and Francis, Boca, Raton, FL, 81-140, 2005

[38] F.J. Valdes-Parada, J.A. Ochoa-Tapia, J. AlvarezRamirez, On the effective viscosity for the DarcyBrinkman equation, Phys. A 385 (2007) 69-79

[39] S. Whitaker, The Method of Volume Averaging, Kluwer, Dordrecht, 1999, p. 173

[40] H. Liu, P.R. Patil, U. Narusawa, On Darcy-Brinkman Equation: Viscous Flow Between Two Parallel Plates Packed with Regular Square Arrays of Cylinders, Entropy 9 (2007) 118-131 